\title{
Amélioration de l'humain et la notion de perfection
}

\section{Jean Martin}

Dr med., membre de la rédaction

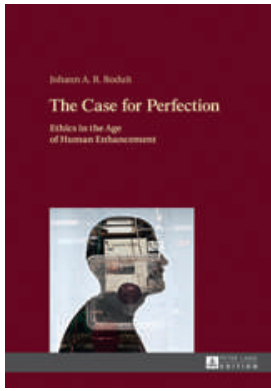

Johann A. R. Roduit The Case for Perfection

Ethics in the Age of Human Enhancement Frankfurt am Main: Peter Lang Edition; 2016.

130 pages. $32 \mathrm{CHF}$ ISBN 978-3-631-67150-4

A propos de l'exercice de ces capacités, Nussbaum donne un exemple interpellant: une personne affamée par manque de nourriture et une personne qui jeûne sont dans une même situation objective s'agissant de leur nutrition, mais n'ont pas la même capacité à la modifier.

jean.martin[at]saez.ch
Johann Roduit, philosophe, est Managing Director du Centre des humanités médicales de l'Institut d'éthique biomédicale de l'Université de Zurich. Il est fort actif depuis plusieurs années dans le domaine bioéthique et anime aussi des évènements TED à Martigny. The Case for Perfection est issu de sa récente thèse de Ph.D. sur les enjeux éthiques de l'amélioration de l'humain (enhancement).

D'abord, l'auteur relève qu'il y a plusieurs définitions possibles du "enhancement»: au sens d'intervention du registre médical qui tend à améliorer/renforcer un trait existant particulier (par exemple chirurgie cosmétique), sans qu'il s'agisse de guérir; au sens d'addition d'une ou des caractéristiques désirables nouvelles (vision quantitative); au sens de changement qualitatif. Cette dernière est celle qu'il adopte, dans une perspective menant à un certain but. Dans cette acception, le traitement d'une maladie peut être vu comme une forme de «enhancement». Etant entendu qu'une question majeure est «amélioration, mais de quel point de vue, en fonction de quoi?». Argumentant qu'il est inévitable de considérer la notion de perfection dans ce débat, l'auteur en distingue différentes conceptions. Il estime que le concept doit satisfaire à plusieurs critères objectifs, sur un mode pluraliste, sans qu'il s'agisse d'avoir une vision fixée de ce que "être humain» devrait signifier. Dans la réflexion, l'image d'un idéal humain devient un point de référence à considérer aux côtés de notions comme l'autonomie, la justice, la sécurité ou l'authenticité.

Roduit mène son travail en exposant les opinions des bio-éthico-conservateurs comme celles des bio-éthicolibéraux, avec entre autres l'objectif de distinguer ce qui sera(it) amélioration vraie de celle qui serait malvenue/indésirable (dis-enhancement). Il relève que les conservateurs, qui s'opposent au «enhancement» et critiquent les libéraux pour leur recherche d'une certaine perfection, soutiennent néanmoins eux-mêmes certains postulats perfectionnistes quant à la «bonne vie».
La thèse de l'auteur est que la "capabilities approach" de la philosophe américaine Martha Nussbaum, spécialiste du développement et de l'éthique qui a travaillé avec Amartya Sen, qui définit dix capacités centrales de la personne*, représente un bon instrument dans la démarche à suivre. Pour être moralement acceptable, l'amélioration de l'humain doit maximiser ces capacités centrales, de manière holistique et harmonieuse. Ceci devrait «maintenir une certaine unité au sein de l'humanité tout en permettant la diversité» - et inclure des garde-fous assurant que l'amélioration en question représente un réel progrès et ne court pas le risque d'une évolution vers le sous-humain. Ici, une remarque montrant un des aspects délicats du «enhancement»: «Théoriquement, on peut imaginer des circonstances dans lesquelles une diminution de l'intelligence pourrait être vue comme une amélioration [admissible], alors que dans d'autres situations cela serait moralement inacceptable.» Well?... (L'auteur ne détaille pas la description d'une telle éventualité.)

Etant entendu qu'une question majeure est «amélioration, mais de quel point de vue, en fonction de quoi?».

Roduit écrit: «J'ai présenté deux visions: l'une nonidéaliste, comparative et rétrospective, l'autre prospective, orientée vers un ou des buts idéaux. A mon avis il faut adopter une approche globale dans laquelle ces deux visions jouent un rôle essentiel. Bien que la seconde fasse l'objet de critiques, elle est nécessaire.» Débattre de la notion de perfection fait partie intégrante de la problématique "enhancement», hyperactuelle, et on peut savoir gré à Roduit de s'être penché en détail sur diverses conceptions possibles, tirant les conclusions à son sens les plus équilibrées. Son texte est en général de nature académique, logiquement puisque issu de sa thèse, et n'échappe pas à certaines répétitions, mais toute personne intéressée suivra sans difficulté sa réflexion, sur un sujet complexe. 